\title{
Efeitos da suplementação com creatina sobre o metabolismo glicêmico: uma revisão sistemática
}

\section{Effects of creatine supplementation on glycemic metabolism: a systematic review}

\author{
Gabriel Elias Ota ${ }^{1, *}$ \\ Camila Souza de Morais ${ }^{1}$ \\ Paula Felippe Martinez ${ }^{1}$ \\ Silvio Assis de Oliveira Júnior ${ }^{1}$
}

\begin{abstract}
Resumo
Introdução: Estudos experimentais demonstraram que a suplementação com creatina promove hiperinsulinemia e/ ou hiperglicemia. Entretanto, outros estudos refutam essa possibilidade, demonstrando que a creatina não interfere ou até melhora a tolerância glicêmica. Objetivo: esclarecer os efeitos de diferentes suplementações com creatina sobre indicadores do metabolismo de glicose e insulina no meio sistêmico e tecidual. Métodos: A pesquisa foi realizada na base de dados PUBMED, SCIELO, SCOPUS e BIREME. Resultados: Na linha experimental, foram encontrados 16 manuscritos e 8 ensaios clínicos. A análise dos trabalhos revelou importantes divergências metodológicas, o que explica os diferentes resultados encontrados entre modelos experimentais. Conclusão: No contexto clínico, a suplementação com creatina, per se, não alterou a homeostase glicêmica; quando associada ao exercício físico, tem repercutido em melhora no metabolismo de glicose.
\end{abstract}

Palavras-chave: creatina, glicose, insulina.

\section{Abstract}

Introduction: While experimental studies have shown that creatine supplementation promotes hyperinsulinemia and/ or hyperglycemia, other studies have refuted this association, since creatine does not interfere with or even improve glycemic tolerance. Objective: clarify the effects of different creatine supplementation on glucose and insulin metabolism indicators in the systemic and tissue environment. Methods: The research was carried out in the database PUBMED, SCIELO, SCOPUS and BIREME. Results: In the experimental line were found 16 manuscripts and 8 clinical trials. The results found methodological differences, which explains the different results found in the experimental model. Conclusion: Clinically, creatine supplementation, per se, did not alter a glycemic homeostasis; however, when associated with exercise, it promoted greater glycemic tolerance.
}

Keywords: creatine, glucose, insulin.
Afiliação dos autores

${ }^{1}$ Universidade Federal de Mato Grosso do Sul, Campo Grande, Mato Grosso do Sul, Brasil

${ }^{\star}$ Autor correspondente

Rua Barão do Rio Branco, 1348, Apartamento 602,Centro, Campo Grande, Mato Grosso do Sil, Brasil. e-mail:ota_gabriel@hotmail.com

Conflito de interesses

Os autores declararam não haver conflito de interesses.

Processo de arbitragem

Recebido: 22/08/2017 Aprovado: 13/08/2018 


\section{Introdução}

Cresce de forma contínua o interesse pela nutrição aplicada ao treinamento desportivo, e essa procura se dá por atletas e praticantes de várias modalidades esportivas com a finalidade de melhorar o desempenho físico, sob a premissa de reposição energética e/ou auxílio na recuperação pós-esforço ${ }^{1}$. Dentre os mais diversos suplementos comercializados, destaca-se a creatina monohidratada ou creatina, como é comumente popularizada e utilizada entre usuários de diferentes faixas etárias ${ }^{2}$. A frequência de uso varia de acordo com a modalidade, sendo mais frequente entre adeptos de treinamento resistido e no fisiculturismo ${ }^{2}$.

A creatina é uma amina nitrogenada, quimicamente conhecida como ácido acético a-metilguanidina. É formada naturalmente pelo fígado e pelos rins, por intermédio dos aminoácidos glicina, arginina e metionina, e pode ser obtida a partir da ingestão de carnes, peixes e outros alimentos de origem animal ${ }^{2}$. No corpo humano, aproximadamente $95 \%$ é armazenada no tecido muscular, enquanto o restante é encontrado no coração, cérebro, pulmões, testículos, fígado e rins $^{2}$. No músculo estriado esquelético, a creatina é relacionada com o processo de contração, servindo-se de substrato para o grupo fosfato, formando a fosfocreatina (CP). Dessa forma, contribui para maior disponibilidade de $\mathrm{CP}$, ampliando a oferta de energia e a ressíntese de trifosfato de adenosina (ATP); assim, a creatina é considerada um recurso energético para atividades de curta duração que necessitam de potência e velocidade ${ }^{2}$.

Embora alguns estudos de revisão $0^{3,4}$ e metanálises ${ }^{5,6}$ tenham documentado os efeitos ergogênicos da suplementação com creatina sobre a musculatura esquelética, algumas evidências sugerem que esse tipo de intervenção acarreta distúrbios no metabolismo glicêmico ${ }^{7-9}$, em razão de estimular a secreção pancreática de insulina. A hipersecreção deste hormônio, a longo prazo, pode resultar em hiperinsulinemia e conduzir a um quadro de resistência à insulina ${ }^{10}$. Sob essas considerações, o presente trabalho de revisão foi proposto para esclarecer os efeitos de diferentes suplementações com creatina sobre indicadores do metabolismo de glicose e insulina no meio sistêmico e tecidual, tendo por base evidências documentadas na literatura.

\section{Métodos}

Para desenvolvimento do estudo, a busca bibliográfica foi realizada em bases eletrônicas de dados (PUBMED, SCIELO, SCOPUS E BIREME) e lista de referências dos artigos identificados. A seleção dos descritores utilizados no processo de revisão foi efetuada mediante consulta ao DECs (descritores de assunto em ciências da saúde da BIREME). Nas buscas para estudos experimentais, utilizou-se os seguintes descritores, em português e inglês: "creatina", "insulina", "glicose" e "ratos". Na busca de estudos clínicos, foram usados os mesmos descritores em português e inglês, retirando-se o termo "ratos" e incluindose os termos: "homens" e "humanos". Recorreu-se aos operadores lógicos "AND", "OR" e "AND NOT" para combinação dos descritores e termos utilizados para rastreamento das publicações. Para seleção dos trabalhos, foi delimitado o período entre Janeiro de 2000 a Julho de 2017.

Como critérios de inclusão, estabeleceu-se a seleção de experimentos com modelo animal e estudos clínicos que tenham investigado efeitos de suplementações com creatina no metabolismo glicêmico e insulinêmico, em nível sistêmico e tecidual. Para triagem de dados em cada bloco de busca, considerando-se, respectivamente, estudos experimentais e clínicos, foram utilizadas tabelas para exposição dos achados bibliográficos, destacando-se as seguintes informações: autor principal, ano de publicação, periódico e base de dados de procedência. Outras tabelas foram constituídas com a listagem de autores e ano, variáveis analisadas, vigência de outras intervenções e principais resultados encontrados.

Após avaliação dos resumos, os estudos que preencheram os critérios de inclusão foram obtidos e lidos na íntegra. Os procedimentos de busca e avaliação das referências foram realizados por dois pesquisadores de forma independente e as divergências foram sanadas por um terceiro investigador.

\section{Resultados}

Considerando-se os trabalhos com modelos animais, inicialmente, foram encontrados um total de 376 artigos, provindos das bases de dados PubMed $(n=341)$, Scielo $(n=7)$, Scopus $(n=21)$ e Bireme $(n=7)$. Após a análise do título e do resumo, foram selecionados 32 artigos, que atenderam aos critérios de inclusão; 344 investigações foram excluídas por não avaliarem respostas de intervenções com creatina sobre 0 metabolismo glicêmico e insulinêmico. Após a leitura completa dos artigos, foram selecionados 14 manuscritos completos, que atenderam a todos os critérios de inclusão pré-estabelecidos. Levando-se em conta os artigos selecionados para leitura completa, mais dois trabalhos foram incluídos, após consulta às seções de referências bibliográficas, totalizando-se 16 manuscritos que foram integrados à presente revisão bibliográfica (Tabela 1).

Entre os 16 manuscritos obtidos, 14 envolveram modelos animais e dois trabalhos foram realizados por meio de ensaios celulares. Considerando-se esse montante amostral, apenas $37,5 \%(n=6)$ do total de trabalhos foram publicados em periódicos nacionais. Em relação ao período em que se baseou a busca, foram encontrados 11 trabalhos $(68,8 \%)$ publicados entre 2000 e 2008; no período subsequente, foram encontradas cinco publicações $(31,2 \%)$ (Tabela 1$)$.

Tabela1

Relação de trabalhos inclusos no processo de revisão bibliográfica, que tratam de modelos animais de experimentação.

\begin{tabular}{|c|c|c|c|}
\hline Autor & Ano & Periódico & Base de Dados \\
\hline $\begin{array}{l}\text { Ferrante } \\
\text { RJ.1 }\end{array}$ & 2000 & Journal of Neuroscience & PubMed \\
\hline Eijnde $B O .^{12}$ & 2001 & Acta Physiologica & $\begin{array}{l}\text { PubMed; } \\
\text { Bireme }\end{array}$ \\
\hline Rooney K. ${ }^{13}$ & 2002 & $\begin{array}{l}\text { Metabolism Clinical and } \\
\text { Experimental }\end{array}$ & $\begin{array}{l}\text { PubMed; } \\
\text { Bireme; Scopus }\end{array}$ \\
\hline Young JC. ${ }^{14}$ & 2002 & Life Science & $\begin{array}{c}\text { PubMed; } \\
\text { Bireme; Scopus }\end{array}$ \\
\hline $\begin{array}{l}\text { Ceddia } \\
\text { RB. }^{15}\end{array}$ & 2004 & The Journal of Physiology & $\begin{array}{l}\text { PubMed; } \\
\text { Bireme }\end{array}$ \\
\hline Ju JS. ${ }^{16}$ & 2005 & $\begin{array}{c}\text { American Journal of } \\
\text { Physiology - Endocrinology } \\
\text { and Metabolism }\end{array}$ & PubMed \\
\hline Eijnde BO. ${ }^{17}$ & 2006 & $\begin{array}{l}\text { International Journal of } \\
\text { Molecular Medicine }\end{array}$ & $\begin{array}{l}\text { Pubmed; } \\
\text { Bireme }\end{array}$ \\
\hline Silva CA. ${ }^{18}$ & 2006 & $\begin{array}{c}\text { Revista Brasileira de Nutrição } \\
\text { Clínica }\end{array}$ & Referências \\
\hline Souza RA. ${ }^{19}$ & 2006 & $\begin{array}{c}\text { Revista Brasileira de Medicina dc } \\
\text { Esporte }\end{array}$ & Scielo; Bireme \\
\hline $\begin{array}{l}\text { Costallat } \\
\text { BL. }^{8}\end{array}$ & 2007 & $\begin{array}{c}\text { Revista Brasileira de Medicina } \\
\text { do Esporte }\end{array}$ & Scielo; Bireme \\
\hline Freire $\mathrm{TO}^{20}$ & 2008 & $\begin{array}{l}\text { Revista Brasileira de Medicina } \\
\text { do Esporte }\end{array}$ & Scielo \\
\hline Careti . $^{21}$ & 2010 & $\begin{array}{c}\text { Cellular Physiology and } \\
\text { Biochemistry }\end{array}$ & PubMed \\
\hline Vaisy $\mathrm{M}^{22}$ & 2011 & $\begin{array}{l}\text { Journal of Nutritional } \\
\text { Biochemistry }\end{array}$ & $\begin{array}{c}\text { PubMed; } \\
\text { Bireme; Scopus }\end{array}$ \\
\hline Nicastro $\mathrm{H}^{9}{ }^{9}$ & 2012 & Amino Acids & $\begin{array}{c}\text { PubMed; } \\
\text { Bireme; Scopus }\end{array}$ \\
\hline Araújo MB. ${ }^{23}$ & 2012 & Motriz & Scielo \\
\hline $\begin{array}{l}\text { Júnior } \\
\text { RCV. }^{24}\end{array}$ & 2014 & $\begin{array}{l}\text { Revista Brasileira de Nutrição } \\
\text { Esportiva }\end{array}$ & Referências \\
\hline
\end{tabular}

Além de autoria e ano de publicação, na Tabela 2, são mostradas informações relativas às amostras utilizadas, protocolos de intervenção e resultados obtidos. Nove trabalhos $(56,25 \%)$ ofertaram creatina na ração, enquanto quatro investigações a utilizaram como suplemento hídrico (25\%); além disso, uma intervenção se baseou em suplemento gelatinoso $(6,25)$. Como intervenção secundária, apenas quatro trabalhos (25\%) utilizaram protocolos de exercício físico. A metodologia para avaliação do metabolismo glicêmico e insulinêmico consistiu de análises do metabolismo sistêmico, presentes em oito trabalhos $(50 \%)$, análises séricas, vigentes em cinco $(31,25 \%)$, e respostas moleculares mediante incubação, pautadas em duas investigações (12,5\%). Considerando-se os resultados encontrados, três trabalhos documentaram a ocorrência de hiperglicemia e/ ou hiperinsulinemia, enquanto outros seis estudos publicaram aumento da tolerância glicêmica e insulinêmica decorrente da suplementação, e sete investigações mostraram nenhuma diferença significativa. 
Tabela 2

Efeitos da suplementação de creatina sobre metabolismo glicêmico e insulinêmico em modelo animal

\begin{tabular}{|c|c|c|c|c|c|}
\hline Autores & Amostra & $\begin{array}{l}\text { Regime de suplementação } \\
\text { com Creatina }\end{array}$ & $\begin{array}{l}\text { Intervenção } \\
\text { Secundária }\end{array}$ & Métodos & Principais Resultados \\
\hline $\begin{array}{l}\text { Rooney et } \\
\text { al. }^{13}\end{array}$ & $\begin{array}{l}\text { Ratos Wistar } \\
\quad(n=16)\end{array}$ & $\begin{array}{c}2 \% \text { de creatina na ração; } \\
\text { durante } \\
2,4 \text { e } 8 \text { semanas; }\end{array}$ & Sem exercício & TTG & $\begin{array}{c}\uparrow \text { Concentração de } \\
\text { insulina e glicose - } 4^{\circ} \\
\text { e } 8^{\circ} \text { semana; } \\
\uparrow \text { Da glicemia no } \\
\text { tempo basal e 15' }\end{array}$ \\
\hline $\begin{array}{l}\text { Costallat et } \\
\text { al. }^{8}\end{array}$ & $\begin{array}{c}\text { Ratos Wistar } \\
\text { ( } \mathrm{n}=24 \text { fêmeas e } 24 \text { machos) }\end{array}$ & $\begin{array}{l}0,4 \mathrm{~g} \text { de creatina para } 30 \mathrm{ml} \text { de } \\
\text { água, durante } 4 \text { semanas. }\end{array}$ & Sem exercício & TTI & $\begin{array}{c}\text { após } 14 \text { dias de } \\
\text { experimento; } \uparrow \mathrm{Da} \\
\text { glicemia em todos os } \\
\text { tempos no } 21^{\circ} \text { e } 28^{\circ} \\
\text { dia; }\end{array}$ \\
\hline $\begin{array}{l}\text { Nicastro et } \\
\text { al. }^{9}\end{array}$ & $\begin{array}{l}\text { Ratos Wistar } \\
\quad(n=24)\end{array}$ & $\begin{array}{c}5 \mathrm{~g} / \mathrm{kg} / \text { dia na água, durante } 7 \\
\text { dias. }\end{array}$ & Sem exercício & $\begin{array}{c}\text { Glicose e Insulina no } \\
\text { Soro }\end{array}$ & $\begin{array}{l}\uparrow \text { Glicose e Insulina no } \\
\text { soro do grupo } \\
\text { Creatina; }\end{array}$ \\
\hline Freire et al. ${ }^{20}$ & $\begin{array}{l}\text { Ratos Wistar } \\
\quad(\mathrm{n}=65)\end{array}$ & $\begin{array}{l}2 \% \text { de creatina na ração, } \\
\text { durante } 4 \text { e } 8 \text { semanas. }\end{array}$ & $\begin{array}{l}\text { Natação - } 4 \text { vezes } \\
\text { por semana }\end{array}$ & TTG & Não houve diferença \\
\hline Vaisy et al. ${ }^{22}$ & $\begin{array}{l}\text { Ratos Wistar } \\
\quad(\mathrm{n}=69)\end{array}$ & $\begin{array}{l}2,5 \% \text { de creatina na ração, } \\
\text { durante } 12 \text { semanas. }\end{array}$ & $\begin{array}{c}\text { Natação - } 5 \text { vezes } \\
\text { na semana. }\end{array}$ & TTG & Não houve diferença \\
\hline $\begin{array}{l}\text { Araújo et } \\
\text { al. }^{23}\end{array}$ & Ratos Wistar $(n=40)$ & $\begin{array}{l}13 \% \text { de creatina na ração, } \\
\text { durante } 7 \text { dias e } 2 \% \text { durante } \\
55 \text { dias; }\end{array}$ & $\begin{array}{l}\text { Exercício em } \\
\text { esteira - } 5 \text { vezes } \\
\text { na semana }\end{array}$ & TTG & $\begin{array}{l}\text { Melhora da tolerância } \\
\text { glicêmica quando } \\
\text { submetidos ao } \\
\text { exercício }\end{array}$ \\
\hline Júnior et al. ${ }^{24}$ & Ratos Wistar $(n=10)$ & $\begin{array}{l}\text { 13\% de creatina na ração, } \\
\text { durante } 10 \text { dias. }\end{array}$ & Sem exercício & TTG; TTI & Não houve diferença \\
\hline Eijnde et al. ${ }^{17}$ & $\begin{array}{l}\text { Ratos Goto-Kakizaki } \\
\qquad(\mathrm{n}=24)\end{array}$ & $\begin{array}{l}2 \% \text { de creatina na ração, } \\
\text { durante } 8 \text { semanas; }\end{array}$ & Sem exercício & $\begin{array}{l}\text { TTG; Insulina no Plasma; } \\
\text { Glicose no sangue }\end{array}$ & $\downarrow$ Insulina plasmática; \\
\hline Eijnde et al. ${ }^{12}$ & $\begin{array}{c}\text { Ratos Wistar } \\
(\mathrm{n}=\text { não relatado })\end{array}$ & $\begin{array}{l}\text { 5\% de creatina na ração, } \\
\text { durante } 5 \text { dias. }\end{array}$ & Sem exercício & $\begin{array}{l}\text { Insulina no Plasma; } \\
\text { Glicose no Sangue }\end{array}$ & Não houve diferença \\
\hline $\begin{array}{c}\text { Silva e } \\
\text { Cancelliero }\end{array}$ & Ratos Wistar $(n=36)$ & $\begin{array}{l}1,6 \mathrm{~g} / \mathrm{kg} / \text { dia de creatina na } \\
\text { água, durante } 7 \text { dias. }\end{array}$ & Sem exercício & Glicose no Plasma & Não houve diferença \\
\hline $\begin{array}{l}\text { Ceddia e } \\
\text { Sweeney }^{15}\end{array}$ & Ratos L6 (Cultura em célula) & $\begin{array}{l}48 \text { horas em solução com } 0,5 \\
\text { mm de creatina }\end{array}$ & Sem exercício & $\begin{array}{l}\text { Células incubadas em } \\
\text { insulina }\end{array}$ & Não houve diferença \\
\hline $\begin{array}{l}\text { Young e } \\
\text { Young }^{14}\end{array}$ & $\begin{array}{l}\text { Ratos Sprague-Dawley } \\
\qquad(\mathrm{n}=8)\end{array}$ & $\begin{array}{l}300 \mathrm{mg} / \mathrm{kg} \text { de creatina na } \\
\text { gelatina, durante } 5 \text { semanas; }\end{array}$ & Sem Exercício & $\begin{array}{l}\text { Músculo } \\
\text { Epitrochlearisincubado } \\
\text { com insulina }\end{array}$ & Não houve diferença \\
\hline Souza et al. ${ }^{19}$ & $\begin{array}{l}\text { Ratos Wistar } \\
\quad(\mathrm{n}=72)\end{array}$ & $\begin{array}{c}5 \mathrm{~g} / \mathrm{kg} \text { de } \mathrm{Cr} \text { (fase de carga - } \\
1 \text { semana); } \\
1 \mathrm{~g} / \mathrm{kg} \text { de } \mathrm{Cr} \text { (fase de } \\
\text { manutenção }-8 \text { semanas) }\end{array}$ & $\begin{array}{l}\text { Natação - } \\
5 \text { vezes por } \\
\text { semana }\end{array}$ & $\begin{array}{l}\text { Glicose no plasma após } \\
\text { teste de carga máxima }\end{array}$ & $\begin{array}{c}\downarrow \text { da glicose } \\
\text { plasmática após teste } \\
\text { de carga máxima. } \\
\text { Melhora na } \\
\text { sensibilidade à insulina }\end{array}$ \\
\hline Ju et al. ${ }^{16}$ & $\begin{array}{l}\text { Ratas Wistar } \\
\quad(n=14)\end{array}$ & $\begin{array}{l}2 \% \text { de creatina na ração, } \\
\text { durante } 3 \text { semanas; }\end{array}$ & Sem exercício & $\begin{array}{c}\text { Músculo } \\
\text { EpitrochlearisIncubado } \\
\text { em insulina; } \\
\text { concentração de GLUT4 }\end{array}$ & $\begin{array}{c}\uparrow \text { na expressão de } \\
\text { Glut4 }\end{array}$ \\
\hline $\begin{array}{l}\text { Ferrante et } \\
\quad \text { al. }^{11}\end{array}$ & $\begin{array}{l}\text { Camundongos transgênicos } \\
\text { com doença de Huntington R6/2 } \\
(\mathrm{n}=50)\end{array}$ & $\begin{array}{l}1,2 \text { ou } 3 \% \text { de creatina } \\
\text { durante } 12 \text { semanas; }\end{array}$ & Sem exercício & TTG & $\begin{array}{c}\text { Melhora na tolerância } \\
\text { a glicose. }\end{array}$ \\
\hline Careti et al. ${ }^{21}$ & Cultura de células & $0,5 \mu \mathrm{M}$ creatina + ribose & Sem exercício & $\begin{array}{c}\text { Western Blot da proteína } \\
\text { AKT/ PKB }\end{array}$ & $\begin{array}{c}\text { Melhora na tolerância } \\
\text { a glicose }\end{array}$ \\
\hline
\end{tabular}
tipo 4.

Levando-se em conta o bloco de trabalhos clínicos, foram encontrados 234 artigos indexados em diferentes bases de dados, incluindo-se PubMed $(n=171)$, Bireme $(n=24)$, Scopus $(n=33)$ e Scielo $(n=6)$. Seguindo-se à leitura do título e do resumo, foram selecionados 20 trabalhos, dentre os quais apenas oito atenderam plenamente aos critérios estabelecidos. Os manuscritos selecionados são apresentados na Tabela 3.

Tabela 3

Relação de trabalhos inclusos no processo de revisão bibliográfica, que tratam de estudos clínicos

\begin{tabular}{|c|c|c|c|}
\hline Autor & Ano & Periódico & Base de Dados \\
\hline Eijnde $\mathrm{BO}^{25}$ & 2001 & Diabetes & PubMed;Bireme \\
\hline Newman $\mathrm{N}^{26}$ & 2003 & $\begin{array}{c}\text { Medicine \& Science in Sports \& } \\
\text { Exercise }\end{array}$ & PubMed; Bireme \\
\hline Derave W. ${ }^{27}$ & 2003 & Journal of Applied Physiology & PubMed;Bireme \\
\hline Van Loon LJ. ${ }^{28}$ & 2004 & Clinical Science & PubMed; Bireme \\
\hline Safdar A. ${ }^{29}$ & 2008 & Physiological Genomics & PubMed; Bireme \\
\hline Gualano B. ${ }^{30}$ & 2008 & Amino Acids & PubMed; Bireme \\
\hline Gualano B. ${ }^{31}$ & 2011 & $\begin{array}{c}\text { Medicine \& Science in Sports \& } \\
\text { Exercise }\end{array}$ & PubMed \\
\hline Alves $\mathrm{CR}^{32}$ & 2012 & Amino Acids & PubMed \\
\hline
\end{tabular}

Do total de artigos selecionados para a presente revisão bibliográfica, $100 \%$ dos trabalhos foram publicados em periódicos internacionais. Considerando-se o período em que se baseou a busca, foram encontrados seis trabalhos (75\%) publicados entre 2000 e 2008; no período subsequente, foram encontradas duas publicações (25\%). Além disso, dois trabalhos (25\%) integraram ensaios clínicos com portadores de diabetes mellitus tipo 2.

O emprego de metodologia para avaliação do metabolismo glicêmico e insulinêmico foi consubstanciado em cinco trabalhos $(62,5 \%)$ e envolveu análises do metabolismo sistêmico, enquanto seis trabalhos $(75 \%)$ incluíram também análises teciduais. Considerando-se os resultados encontrados, cinco manuscritos apresentaram melhora na tolerância glicêmica e três estudos não reportaram efeito algum (Tabela 4).

\section{Discussão}

O presente trabalho foi proposto para descrever os efeitos de diferentes intervenções com creatina sobre indicadores do metabolismo de glicose e insulina no meio sistêmico e tecidual. Partindo-se de evidências da literatura, apenas três trabalhos $(18,75 \%)$ demonstraram que a suplementação com creatina em modelo animal pode conduzir a hiperinsulinemia e/ ou hiperglicemia e possível resistência à insulina. Contrariamente, cinco estudos clínicos $(62,5 \%)$ e cinco experimentais $(31,3 \%)$ revelaram que a suplementação com creatina promove melhora na tolerância glicêmica. Três investigações clínicas $(37,5 \%)$ e outras sete pesquisas experimentais $(43,8 \%)$ mostraram nenhum efeito da suplementação com creatina sobre metabolismo glicêmico.

No contexto experimental, as presentes divergências são, provavelmente, decorrentes de variações entre protocolos de suplementação com creatina; com razão, não há consenso em relação aos efeitos dose-dependentes ou ainda tempodependentes. No primeiro caso, as formas de administração têm sido variáveis entre veículo sólido, solução aquosa e, inclusive, gelatina, o que confere importante imprecisão à determinação de dosagem. Considerando-se somente os protocolos de ração, as dosagens tem se situado entre $1 \%$ a $13 \%$ (Tabela 2), não havendo relação direta entre a quantidade ingerida e os efeitos obtidos. Enquanto suplementação com $2 \%$ de creatina mostrou provocar hiperglicemia e hiperinsulinemia ${ }^{13}$, intervenções com $13 \%$ não alteraram ${ }^{24}$ ou, inclusive, melhoraram a tolerância metabólica $^{23}$. 
Tabela 4

Efeitos da suplementação de creatina sobre metabolismo glicêmico e insulinêmico em humanos.

\begin{tabular}{|c|c|c|c|c|c|}
\hline Autores & Amostra & Regime de suplementação & Intervenção Secundária & $\begin{array}{c}\text { Teste de Glicose e } \\
\text { Insulina }\end{array}$ & Principais Resultados \\
\hline Eijinde et al. ${ }^{25}$ & $\begin{array}{l}13 \text { homens; } 9 \\
\text { mulheres }\end{array}$ & $\begin{array}{l}1-2 \text { semanas: } 20 \mathrm{~g} / \mathrm{dia} \\
3-5 \text { semanas: } 15 \mathrm{~g} / \mathrm{dia} \\
6-12 \text { semanas: } 5 \mathrm{~g} / \mathrm{dia}\end{array}$ & $\begin{array}{l}\text { Programa de reabilitação } \\
\text { durante } 10 \text { semanas }-3 x \\
\quad \text { por semana }\end{array}$ & $\begin{array}{l}\text { Análise do } \\
\text { conteúdo de } \\
\text { GLUT4 do músculo } \\
\text { vasto lateral }\end{array}$ & $\begin{array}{l}\uparrow \text { do conteúdo de } \\
\text { GLUT4 quando } \\
\text { associado ao } \\
\text { exercício físico }\end{array}$ \\
\hline $\begin{array}{l}\text { Newman et } \\
\text { al. }^{26}\end{array}$ & 17 jovens: & $\begin{array}{c}\text { Fase de Carga: } 5 \text { gramas; } \\
\text { Fase e Manutenção: } 3 \text { gramas } \\
\text { Grupo Creatina: } 5 \mathrm{~g} / \text { dia (imobilizacão) e }\end{array}$ & Sem exercício & $\begin{array}{l}\text { GTT; Insulina em } \\
\text { jejum }\end{array}$ & Não houve diferença \\
\hline Derave et al. ${ }^{27}$ & $\begin{array}{l}26 \text { homens; } 7 \\
\text { mulheres: }\end{array}$ & $\begin{array}{c}\text { Grupo Creatina: } 5 \mathrm{~g} / \text { dia (imobilização) e } \\
\text { 2,5g/dia (reabilitação); Grupo Creatina + } \\
\text { Proteína: } 15 \mathrm{~g} / \text { dia (imobilização) e } 2,5 \mathrm{~g} / \text { dia } \\
\text { com } 40 \mathrm{~g} \text { proteína e } 6,7 \mathrm{~g} \text { de amino ácido } \\
\text { durante a reabilitação. }\end{array}$ & $\begin{array}{l}\text { Programa de reabilitação } \\
\text { por } 6 \text { semanas }\end{array}$ & GTT; GLUT4 & $\begin{array}{l}\uparrow \text { do conteúdo de } \\
\text { GLUT4; } \\
\text { melhora tolerância a } \\
\text { glicose }\end{array}$ \\
\hline $\begin{array}{l}\text { Van Loon et } \\
\text { al. }^{28}\end{array}$ & 19 sujeitos & $\begin{array}{c}\text { Creatina: fase de carga }(20 \mathrm{~g} / \text { dia por } 5 \\
\text { dias, fase manutenção ( } 2 \mathrm{~g} / \mathrm{dia} \text { por } 37 \\
\text { dias); Placebo: maltodrexina na mesma } \\
\text { concentração. }\end{array}$ & Sem exercício & $\begin{array}{l}\text { Insulina em jejum; } \\
\text { GLUT4 }\end{array}$ & Não houve diferença \\
\hline Safdar et al. ${ }^{29}$ & 12 jovens & $\begin{array}{l}\text { Creatina: fase carga }(20 \mathrm{~g} / \text { diapor } 3 \text { dias); } \\
\text { fase de manutenção ( } 5 \mathrm{~g} / \text { dia por } 7 \text { dias) } \\
\text { Placebo: } 75 \mathrm{~g} \text { de dextrose }\end{array}$ & Sem exercício & GLUT4 & Não houve diferença \\
\hline Gualano et al. ${ }^{30}$ & 22 sujeitos & $\begin{array}{c}\text { Creatina: } 10 \mathrm{~g} / \text { dia por } 3 \text { meses; } \\
\text { Placebo: Dextrose }\end{array}$ & Exercício aeróbio moderado & $\begin{array}{l}\text { GTT; Insulina em } \\
\text { jejum. HOMA. }\end{array}$ & $\begin{array}{l}\text { Melhora tolerância a } \\
\text { glicose quando } \\
\text { associada ao } \\
\text { exercício }\end{array}$ \\
\hline Gualano et al. ${ }^{31}$ & $\begin{array}{l}25 \text { sujeitos com } \\
\text { diabetes tipo } 2\end{array}$ & $\begin{array}{l}\text { Creatina }(5 \mathrm{~g} / \mathrm{dia}) \\
\text { Placebo: dextrose }\end{array}$ & $\begin{array}{l}\text { Exercício aeróbio moderado } \\
\text { e treinamento de resistência }\end{array}$ & $\begin{array}{c}\text { GTT; GLUT4 e } \\
\text { insulina em jejum }\end{array}$ & $\begin{array}{l}\text { Melhora no controle } \\
\text { glicêmico; Sem } \\
\text { alteração do } \\
\text { conteúdo de GLUT4 }\end{array}$ \\
\hline Alves et al. ${ }^{32}$ & $\begin{array}{l}25 \text { sujeitos com } \\
\text { diabetes tipo } 2\end{array}$ & $\begin{array}{c}\text { Creatina: }(5 \mathrm{~g} / \mathrm{dia}) \\
\text { Placebo: dextrose por } 12 \text { semanas }\end{array}$ & $\begin{array}{l}\text { Exercício aeróbio moderado } \\
\text { e treinamento de resistência }\end{array}$ & GLUT4; AMPK & $\begin{array}{l}\text { Sem alteração do } \\
\text { conteúdo de GLUT4; } \\
\text { Aumento da } \\
\text { expressão da AMPK- } \\
\alpha\end{array}$ \\
\hline
\end{tabular}

da proteína cinase ativada por AMP.

Os efeitos tempo-dependentes revelam relação mais direta com as respostas metabólicas. Nesse contexto, intervenções com até oito semanas ${ }^{8,9,13}$ sustentam que a suplementação com creatina provoca anormalidades do metabolismo glicêmico e insulinêmico. Já evidências procedentes de protocolos com maior duração ${ }^{14,22,23}$, além de relatos clínicos, demonstraram que a intervencão pouco afeta a homeostase glicêmica e hormonal ${ }^{26,28,29}$. Clinicamente, inclusive, a prática do exercício físico, mais do que a creatina, demonstrou ser fator chave para alterar o controle glicêmico. Portanto, pode-se inferir que alterações do quadro metabólico são mais prováveis em intervenções agudas de creatina. Rooney et al. ${ }^{13}$ encontraram hiperinsulinemiaà partir da quarta semana semana de experimentação perdurando até a oitava semana. No $14^{\circ}$ dia de experimento, Costallat et al. ${ }^{8}$ verificaram hiperglicemia em jejum e, a partir do $21^{\circ}$ e no $28^{\circ}$ dia, tal achado foi acompanhado por menor tolerância glicêmica. Nicastro et al. ${ }^{9}$ também encontraram elevados valores de glicose, indicando hiperglicemia. Esses achados sugerem que a suplementação com creatina é capaz de induzir a uma maior secreção de insulina, provocando hiperinsulinemia/ hiperglicemia, que podem desencadear distúrbios metabólicos como resistência à insulina, principalmente, em protocolos experimentais breves. As repostas verificadas em estudos clínicos não reproduziram resultados similares. Em recente revisão, Pinto et al. ${ }^{33}$ também constataram discrepâncias entre investigações com modelos animais, culminando em possíveis dificuldades de comparação e inferência translacional para estudos clínicos.

De modo geral, apesar da glicose configurar o principal agente incitador de secreção de insulina pelas células $\beta$ pancreáticas, diferentes fatores podem afetar a secreção de insulina, incluindo-se agentes inflamatórios, fármacos, ácidos graxos e aminoácidos, como a arginina ${ }^{34}$, um dos componentes da estrutura molecular de creatina. O processo de estimulação da insulina pela arginina consiste em seu transporte para o interior de células $\beta$ pancreáticas, levando à despolarização da membrana, o que induz abertura de canais de cálcio dependentes de voltagem e, consequentemente, estimula a secreção de insulina ${ }^{35}$. Barbosa et al. ${ }^{36}$ administraram arginina a ratos durante quatro semanas e os resultados demonstraram uma possível resistência à insulina, sustentada pela alta concentração sérica do hormônio associada a menor taxa de decaimento glicêmico no teste de tolerância à insulina. Em outro estudo, Barbosa et al. ${ }^{37}$ investigaram a expressão de protéinas envolvidas na sinalização da insulina no músculo esquelético, tecido adiposo e no fígado de ratos suplementados com arginina por quatro semanas. Embora a expressão de subunidades $\beta$ do receptor sarcolemal de insulina (IR) não tenha sido afetada, o conteúdo dos substratos do IR (IRS 1/2) e do peptídeo AKT, com localização pós-receptor, mostraram-se reduzidos nos tecidos analisados, indicando prejuízo na sinalização de insulina em localização pós-receptor. Além disso, as células $\beta$ pancreáticas são também sensíveis à alta concentração de adenosina trifosfato (ATP) e a suplementação com creatina promove um aumento energético na célula aumentando a razão de adenosina trifosfato por adenosina difosfato (ATP/ADP), o que pode estimular o processo de liberação de insulina ${ }^{38}$.

Sob essas considerações, embora algumas pesquisas experimentais tenham demonstrado que a suplementação com creatina seja capaz de induzir hiperinsulinemia e/ ou hiperglicemia, a maioria dos estudos não mostraram efeitos da intervenção e, inclusive, há relatos de melhora no metabolismo glicêmico. Em contraste, no contexto clínico, os achados são mais homogêneos e têm culminado, em grande parte dos estudos, em melhora do metabolismo de glicose e insulina, principalmente na vigência de protocolos de exercício físico $^{25,27,30-32}$. Portanto, conforme Pinto et al. $^{33}$, deve-se ter cautela ao extrapolar os resultados em modelos animais para área clínica.

Assim sendo, mais estudos são necessários para esclarecer seu efeito no metabolismo de insulina e glicose. Em especial, torna-se necessário que futuras investigações com modelos experimentais se voltem para os efeitos metabólicos e teciduais de diferentes protocolos de suplementação com creatina, em relação à dosagem e tempo de intervenção. Dessa forma, será possível obter-se melhor esclarecimento das semelhanças e diferenças entre respostas de experimentos animais e estudos clínicos.

\section{Conclusão}

Enquanto as evidências de experimentos com modelos animais revelam-se conflitantes e sem consenso em relação aos efeitos de suplementações com creatina, os achados clínicos demonstram que essas intervenções não resultam em alterações do metabolismo glicêmico e insulinêmico.

\section{Referências}

1. Molinero $O$, Márquez $S$.Use of nutritional supplements in sports: risks, knowledge, and behavioural-related factors. Nutr Hosp. 2009;24(2):128-34.

2. Terjung R, Clarkson $P$, Eichner ER, Greenhaff $P$, Hespel $P$, Israel $R$, et al. The physiological and health effects of oral creatine supplementation. Med Sci Sports. 2000;32(3):706-17. 
3. Gualano B, Grinowitsch C, Seguro A, Lancha A. A suplementação de creatina, prejudica a função renal?. Rev. Bras Med Esporte. 2008;14(1):6873.

4. Gualano B, Acquesta FM, Ugrinowitsch C, Tricoli V, Serrão JC, Lancha $\mathrm{AH}$. Effects of Creatine Supplementation on Strength and Muscle Hypertrophy: Current Concepts. Rev Bras Med Esporte. 2010;16(3):21923.

5. Branch JD. Effect of creatine supplementation on body composition and performance: a meta-analysis. Int $\mathrm{J}$ Sport Nutr Exerc Metab. 2003;13(2):198-226.

6. Dempsey RL, Mazzone MF Meurer LN Does oral creatine supplementation improve strength? A meta-analysis. J Fam Pract. 2002;51(11):945-51.

7. Rooney K, Bryson J, Phuyal J, Denyer G, Caterson I, Thompson C. Creatine supplementation alters insulin secretion and glucose homeostasis in vivo. Metabolism. 2002;51(4):518-22.

8. Costallat BL, Miglioli L, Silva PAC, Novo NF, Duarte JLG. Resistência à insulina com a suplementação de creatina em animais de experimentação. Rev Bras Med Esporte. 2007:13(1):22-26.

9. Nicastro H, Gualano B, Moraes WM, Salles PV, Luz CR, Santos CA, et al. Effects of creatine supplementation on muscle wasting and glucose homeostasis in rats treated with dexamethasone. Amino Acids. 2012;42(5):1695-701

10. Ferranini E, Natali A, Bell P, Cavallo-Perin P, Lalic N, Mingrone G. Insulin resistance and hypersecretion in obesity. European Group for Study of Insulin Resistance (EGIR). J Clin Invest. 1997;100(5):1166-73.

11. Ferrante RJ, Andreassen OA, Jenkins BG, Dedeoglu A, Kuemmerle S, Kubilus $\mathrm{JK}$, et al. Neuroprotective effects of creatine in a transgenic mouse model of Hutington's disease. J Neurosci, 2000;15;20(12):4389-97.

12. Eijnde BO, Richter EA, Henquin JC, Kiens B, Hespel P. Effect of creatine supplementation on creatine and glycogen contente in rat skeletal muscle. Acta Physiol Scand. 2001;17(12):169-76.

13. Rooney K, Bryson J, Phuyal J, Denyer G, Caterson I, Thompson C. Creatine supplementation alters insulin secretion and glucose homeostasis in vivo. Metabolism. 2002:51(4):518-22.

14. Young JC, Young RE. The effect of creatine supplementation on glucose uptake in rat skeletal muscle. Life Science. 2002; 71(15):1731-37.

15. Ceddia RB, Sweenet G. Creatine supplementation increases glucose oxidation and AMPK phosphorylation and reduces lactate production in L6 rat skeletal muscle cells. J physiol. 2004;1(555):409-21.

16. Ju J, Smith JL, Oppelt PJ, Fisher JS. Creatine feeding increases GLUT4 expression in rat skeletal muscle. Am J Physiology Endocrinol Metab. 2005; 288(2)E347-52.

17. Eijnde BO, Jijakli $\mathrm{H}$, Hespel $\mathrm{P}$, Malaisse WJ. Creatine supplementation increases soleus muscle creatine contente and lowers the insulinogenic index in an animal modelo f inherited type 2 diabetes. Int $\mathrm{J} \mathrm{Mol} \mathrm{Med.} \mathrm{2006;}$ 17(6): $1077-84$

18. Silva $\mathrm{CA}$, Cancelliero KM. Efeito da suplementação oral com creatina no músculo esquelético de membro imobilizado de ratos. Rev Bras Nutr Clin 2006; 21(1):17-22.

19. Souza RA, Santos RM, Osório RAL, Cogo JC, Júnior ACGP, Martins RABL, et al. Influência da suplementação aguda e Crônica de creatina Sobre as concentrações sanguíneas de glicose e lactato de ratos Wistar. Rev. Bras Med Esporte. 2006;1(6):361-5.

20. Freire TO, Gualano B, Leme MD, Polacow VO, Lancha AHJ. Efeitos da Suplementação de Creatina na Captação de Glicose em Ratos Submetidos ao Exercício Físico. Rev. Bras Med Esporte. 2008;14(5):4315.

21. Caretti A, Bianciardi P, Sala G, Terruzzi C, Lucchina F, Samaja M. Supplementation of creatine and ribose prevents apoptosis in ischemic cardiomyocytes. Cell Physiol Biochem. 2010;26(6):831-8.
22. Vaisy M, Szlufcik K, Bock K, Eijnde BO, Proeyen KV, Verbeke K, et al. Exercise-induced, but not creatine-induced, decrease in intramyocellular lipid contente improves insulin sensitivity in rats. J Nutr Biochem. 2011;22(1):1178-85

23. Araújo MB, Vieira RC, Moura LP, Ribeiro C, Costa M, Rodrigo AD, et al. O metabolismo de creatina é alterado devido ao modo como é administrada. Rev. Bras. Nutr. Esportiva. 2012;6(34):315-24

24. Júnior RCV, Araújo MC, Júnior MC, Dalia RA, Voltarelli FA. O uso de creatina pico não altera a homeostase glicêmica e diminui a ingestão alimentar de ratos. Rev. Bras. Nutr. Esportiva. 2014;8(47):307-15.

25. Eijnde BO, Urso B, Richter EA, Greenhaff PL, Hespel P. Effect of ora creatine supplementation on human muscle GLUT4 protein content after immobilization. Diabetes. 2001;50(1):18-23.

26. Newman JE, Hargreaves M, Garnham A, Snow RJ. Effect of creatine ingestion on glucose tolerance and insulin sensitivity in men. Med Sci Sports Exerc. 2003;35(1):69-74

27. Derave W, Eijnde BO, Verbessem P, Ramaekers M, Leemputte MV, Richter EA, et al. Combined creatine and protein supplementation in conjunction with resistance training promotes muscle GLUT-4 content and glucose tolerance in humans. J. Appl. Physiol. 2003;94(5):1910-6.

28. Van Loon JC, Murphy R, Oosterlaar AM, Cameron-Smith D, Hargreaves $\mathrm{M}$, Wagen,akers et al. Creatine supplementati. on increases glycogen storage but not GLUT-4 expression in human skeletal muscle. Clin Sci. 2004;106(1):99-106

29. Safdar A, Yardley NJ, Snow R, Melov R, Tarnapolsky MA. Global and targeted gene expression and protein content in skeletal muscle of young men following shot-term creatine monohydrate supplementation. Physio Genomics. 2008:32(2):219-28.

30. Gualano B, Novaes RB, Artioli CG, Freire TO, Coelho DF, Scagliusi FB, et al. Effects of creatina supplementation on glucose tolerance and insulin sensitivity in sedentary healthy males undergoing aerobic training. Amino Acids. 2008;34(2):245-50.

31. Gualano B, Painneli VS, Roschel H, Artioli GG, Neves M Jr, Pinto AL, et al. Creatine in type 2 diabetes: a randomized, double-blind, placebo-controlled trial. Med Sci Sports Exerc. 2011; 43(5):770-8.

32. Alves CR, Ferreira JC, Siqueira-Filho MA, Carvalho CR, Lancha Jr, Gualano B. Creatine - induced glucose uptake in type 2 diabetes: a role for ampk - a? Amino Acids. 2012;43(4):1803-7.

33. Pinto CL, Botelho PB, Pimentel GD, Campos-Ferraz PL, Mota JF. Creatine supplementation and glycemic control: a systemic review. Amino Acids. 2016,48(9):2103-29

34. Rang HP, Dale MM, Ritter JM. Rang \& Dale's Farmacologia. Rio de Janeiro: Elsevier; 2008

35. Sener A, Best LC, Yates AP, Kadiata MM, Olivares E, Louchami K, et al Stimulus-secretion coupling of arginine-induced insulin release: comparasion between the cationic amino acid and its methyl ester. Endocrine. 2000;13(3):329-40.

36. Barbosa TC, Poyares LL, Machado UF, Nunes MT Chronic ora administration of arginine induces $\mathrm{GH}$ gene expression and insulin resistance. Life Sci. 2006;79(15):1444-9.

37. Barbosa TC, Carvalho JEN, Poyars LL, Bordin S, Machado UF, Nunes MT Potential role of growth hormone in impairment of insulin signaling in skeletal muscle, adipose tissue, and liver of rats chronically treated with arginine. Endocrinology. 2009;150(5):2080-6.

38. Matschinsky FM. Banting Lecture 1995: a lesson in metabolic regulation inspired by the glucokinase glucose sensor paradigm. Diabetes. 1996;45(2):223-41. 